\title{
Transformative Wissenschaft als honest broker? Das passt!
}

\author{
Reaktion auf S. Strunz, E. Gawel in GAIA 26/4 (2017): Transformative \\ Wissenschaft - eine kritische Bestandsaufnahme der Debatte
}

Transformative Science as an Honest Broker? It's Possible! | GAIA 27/1 (2018): 113-116

Keywords: normativity, policy advice, positivism, science-policy interface, transformative science

Armin Grunwald

S ebastian Strunz und Erik Gawel (2017) haben jüngst in GAIA die Debatte um die transformative Wissenschaft wieder aufgenommen. Das ist verdienstvoll, hatte die Auseinandersetzung doch allerlei „lose Enden“ hinterlassen (Strohschneider 2014, Grunwald 2015, Schneidewind 2015, Rohe 2015, von Wissel 2015, Kläy und Schneider 2015). Einen Abschluss der Debatte versprechen die Autoren klugerweise nicht. Im Gegenteil verweisen sie mit Recht darauf, dass hinter dem akuten Disput um transformative Wissenschaft einige der großen Fragen zum Wissenschaftsverständnis stehen, wie sie etwa den Positivismusstreit geprägt haben (dazu Gethmann und Sander 1999). Fragen dieses Typs sind nicht ein für alle Mal beantwortbar, sondern haben ihren Wert als Medium der wissenschaftlichen Selbstverständigung gerade darin, bestimmte Debatten immer wieder neu anzuregen. So gesehen ist es zwar wichtig, vor Hybris zu warnen und die Praxis transformationsorientierter Forschung konkret in den Blick zu nehmen (Jaeger-Erben et al. 2018). Gleichzeitig jedoch verlangen die grundsätzlichen Fragen nach weiterer Klärung. Das sei im Folgenden anhand der zentralen Argumente von Strunz und Gawel versucht.

\section{Honest broker oder issue advocate - ist das hier die Frage?}

Die beiden Autoren konstatieren ein gewisses Lavieren unterschiedlicher Stellungnahmen zur transformativen Wissenschaft (TW) und stellen diese vor eine klare Entweder-oder-Frage (Strunz und Gawel 2017, S. 322). Sie spitzen ihre kritische Bestandsaufnahme auf einen zentralen Punkt zu: Versteht sich transformative Wissenschaft nun als honest broker oder als issue advocate (Pielke 2007)?

Strunz und Gawel betonen die Risiken eines tätigen Engagements für die Transformation zur Nachhaltigkeit als issue advocate, das sie in die Nähe des Lobbyismus rücken (S. 323). Für eine transformative Wissenschaft, die Nachhaltigkeit nicht distanziert erforscht, sondern propagiert, befürchten sie Verlust von Glaub- würdigkeit und Qualität der Forschung (S. 324). Normative Orientierung könne den analytischen Blick auch „trüben“.

In der Verpflichtung der transformativen Wissenschaft auf die Rolle des honest broker gehe ich voll und ganz mit. Die von Strunz und Gawel zitierten Passagen aus meinem früheren Beitrag (Grunwald 2015) gehen genau in diese Richtung. Es bleiben aber zwei Fragen offen. Die erste betrifft die Rolle normativer Argumentation. Wenn Strunz und Gawel zwar explizit einen radikalen Positivismus ablehnen (S. 324), so beschränken sie Wissenschaft doch auf die Seite des Analytischen und Empirischen (S. 324f.). Hier werde ich Widerspruch anmelden. Zweitens halte ich die vorgenommene Bestimmung des honest broker für artifiziell: Wenn transformative Wissenschaft als honest broker unterschiedliche Vorstellungen über Nachhaltigkeit und Transformation nur moderieren dürfte (S. 322), wäre die transformative Wissenschaft in dem, was sie praktisch will (Schneidewind et al. 2016), sozusagen kastriert. Ich werde demgegenüber zu zeigen versuchen, dass das engagierte Verfolgen eines Transformationsinteresses unter bestimmten Bedingungen mit der Rolle des honest broker durchaus vereinbar ist.

\section{Wissenschaft und der normative Diskurs}

Die Rolle des issue advocate machen Strunz und Gawel zunächst an Einmischungen in politische Fragen fest. Aber auch die Propagierung bestimmter inhaltlicher Aspekte der Nachhaltigkeit stellen sie in diesen Rahmen. Danach dürften zum Beispiel Wis- 
senschaftler(innen), die sich für eine dezentrale Energieversorgung einsetzen, nicht die Rolle des honest broker beanspruchen (S. 323). Das ist eine weitreichende These. Fallen Wissenschaftler(innen), die sich trauen, zur Energiewende Stellung zu nehmen, aus der Rolle eines honest broker heraus und werden $\mathrm{zu}$ Lobbyisten einer partikularen Position? Wenn ja, wäre dies eine fatale Alternative: Mund halten oder Lobbyist sein.

Die Autoren geben kein explizites Argument für ihre These an. Ihre Äußerungen zum Verhältnis honest broker und issue advocate lassen sich am Beispiel zentrale versus dezentrale Energieversorgung folgendermaßen erschließen: Mit hoher Plausibili- gieversorgung, können immer mit Gegenargumenten bezweifelt werden. Ob die jeweils vorgebrachten Argumente gut sind, muss sich im Diskurs erweisen (Gethmann und Sander 1999). Interessen jedoch lassen sich nicht wegargumentieren, sondern bleiben Interessen. Das normative Argumentieren für starke Nachhaltigkeit beispielsweise ist keine Interessenvertretung für starke Nachhaltigkeit, sondern stellt eine Position im Diskurs dar, an der man sich reiben kann - und zwar mit Argumenten und nicht weil man andere Interessen hat. Hier werden Interessen und Präferenzen mit der Suche nach guten Argumenten vermischt, oder mit Habermas (1973): Handlungs- und Diskursebene werden nicht un-

\section{Wenn Wissenschaftler(innen) glauben, gute Gründe zu haben, dass Nachhaltigkeit normativ nicht beliebig ist, sondern in dieser oder jener Richtung ausbuchstabiert werden sollte, sollen sie es dann schamhaft verschweigen, um nicht zum Lobbyisten eigener Interessen abgestempelt zu werden?}

tät wird man auf der Basis von Fakten weder empirisch noch analytisch beweisen können, ob ein dezentrales System wissenschaftlich besser als ein zentrales ist oder ob eine bestimmte Mischung optimal ist. Wenn sich Wissenschaftler(innen) trotzdem für eine dezentrale Versorgung aussprechen, kann daher eine solche Position nicht im Rahmen des empirisch-analytischen Wissenschaftsverständnisses legitimiert werden. Nach Strunz und Gawel bleibt dann nur, solche Wissenschaftler(innen) als Lobbyisten dezentraler Energieversorgung und damit als interessegeleitete issue advocates zu sehen. Der Konflikt zwischen Vertretern eines zentralen und dezentralen Energiesystems wird als Interessenkonflikt begriffen. Interessenkonflikte freilich können durch einen honest broker in der Tat nur moderiert werden: Wissenschaft hat kein Mandat, sich autoritativ in diese einzumischen.

Dabei wird jedoch ausgeblendet, dass es auch andere als empirische oder analytische Gründe für eine dezentrale Energieversorgung geben könnte. So könnte eine normativ-politikwissenschaftliche oder ethisch motivierte Argumentation auf eine gleichmäßigere Machtverteilung in Wirtschaft und Gesellschaft durch dezentrale Energieversorgung hinweisen. Oder unter Vorsorgeaspekten könnte eine dezentrale Versorgung als resilienter angesehen werden, weil sie Insellösungen im Falle des Blackouts erleichtert (Petermann et al. 2011). Offenkundig sehen Strunz und Gawel jedoch solche normativen Argumentationsmuster als wissenschaftlich unzulässig an, dafür vielmehr als Ausdruck unterschiedlicher Interessen.

Hier liegt jedoch ein Kategorienfehler vor. Die Annahme, dass normative Argumentation die „legitime Pluralität von Einzelinteressen im demokratischen Prozess“ (Strunz und Gawel 2017, S. 323) aushebeln könne, ist eine Vermischung unterschiedlicher Ebenen. Normativ begründete Aussagen, sei es zur starken Nachhaltigkeit (Ott und Döring 2004) oder zu einer dezentralen Ener- terschieden. Die legitime Artikulation von Interessen auf der Handlungsebene ist zu unterscheiden von der Vorbringung normativer (oder anderer) Argumente auf der Diskursebene. Durch Interventionen auf der Diskursebene kann unmöglich die Artikulation von Interessen delegitimiert werden.

Ich frage einmal zurück: Wie soll denn beispielsweise an der Bedeutung nachhaltiger Entwicklung gearbeitet werden (Grunwald 2016), wenn nicht auch normative Erwägungen ausgetauscht und im wissenschaftlichen, normativen sowie gesellschaftlichen Diskurs in Bezug auf ihre Prämissen und Implikationen geprüft werden? Wenn Wissenschaftler(innen) glauben, gute Gründe zu haben, dass Nachhaltigkeit normativ nicht beliebig ist, sondern in dieser oder jener Richtung ausbuchstabiert werden sollte, sollen sie es dann schamhaft verschweigen, um nicht zum issue advocate und Lobbyisten eigener Interessen abgestempelt zu werden?

Vielmehr ist doch das Gegenteil wünschenswert: inhaltliche Positionierungen auch in normativer Hinsicht, um Kritik und Gegenpositionen zu motivieren und einen normativen Disput über diese Fragen zu entfachen. Nur solche Dispute, in denen normative Positionierungen explizit gemacht, transparent vorgebracht und in die Debatte eingebracht werden, bringen uns weiter. Ich wüsste nicht, warum solche Interventionen nicht in Einklang mit der Rolle des honest broker stehen sollen - immer natürlich vorausgesetzt, dass mit Normativität argumentationsgeleitet, reflektiert und transparent umgegangen wird. Der normative Disput auf der Diskursebene ist der Zwilling des analytisch-empirischen wissenschaftlichen Disputs über Fakten-, Gesetzes- oder Erklärungswissen. Er ist ein Ausprobieren normativer Argumente im Feuer der Auseinandersetzung zum Zweck des Lernens. Dadurch wird beileibe nicht die legitime Pluralität der Interessen gefährdet. Normative Argumente zu vertreten ist kategorial verschieden von Lobbyismus in eigener Sache. 


\section{Der honest broker in der Transformation}

Wissenschaft als honest broker ist auf die Vielfalt von Optionen verpflichtet und soll sie befördern statt auf eine als wünschenswert oder optimal dargestellte Option zu reduzieren (Pielke 2007). ${ }^{1}$ Damit scheint zunächst trotz der wissenschaftstheoretischen Einwände die Diagnose von Strunz und Gawel plausibel: Es passt nicht zusammen, mit normativen Gründen im Rücken engagiert für eine Nachhaltigkeitstransformation einzutreten und in der Rolle des honest broker gleichsam zurückgelehnt eine Moderatorenrolle einzunehmen, in der man nicht substanziell Stellung zu einzelnen Optionen nehmen darf. Tätiges Engagement verträgt sich nicht mit distanzierter Beobachtung, aktive Einmischung nicht mit bloßer Moderation, Lobbyismus für eine bestimmte Lösung nicht mit der Offenheit demokratischer Aushandlung.

Natürlich kommt jetzt ein „aber“, denn so klar ist die Sache bei näherem Hinsehen doch nicht. Zwei Beobachtungen zum Verständnis des honest broker weichen das scheinbar so harte Argument von Strunz und Gawel auf: 1. keine Option wird entwickelt, die nicht durch Argumente für ihre Eignung motiviert ist; 2. bereits das Hinzufügen von Optionen ist nicht rein analytisch-empirisch, sondern schon in sich transformativ.

Zu Punkt 1: Wer Optionen entwickelt, hat auch Argumente, warum er/sie den schon vorhandenen Optionen neue hinzufügt. Ein(e) Wissenschaftler(in), der/die Optionen für die Nachhaltigkeitstransformation entwickelt, sich aber weigert, Argumente zu bringen, warum seine/ihre Option in irgendeiner Hinsicht gut mehr als Moderation. Und wenn die Argumente sich im Diskurs als so gut erweisen, dass sie auf Zustimmung stoßen und vielleicht sogar den betreffenden Optionen die Chance auf Umsetzung in der Praxis geben - dann ist Transformation erfolgt. Also ist die Rolle des honest broker als Optionenentwickler verträglich mit einem transformativen Interesse - wenn es gelingt, entsprechend gute Argumente für die Transformation und die Rolle der dafür entwickelten Optionen darin zu mobilisieren.

Zu Punkt 2: Etwas direkter: Oft ist bereits das In-die-Welt-Setzen neuer Optionen eine transformative Leistung. Denn die neue Option, etwa ein neues Geschäftsmodell zur schnelleren Umsetzung von effizienterer Wärmenutzung in Gebäuden, verändert allein dadurch die Welt, dass sie öffentlich gemacht wird. Illustrativ ist die Analogie zur Technik: Bereits das In-die-Welt-Bringen neuer technischer Optionen verändert die jeweilige Konstellation. Das ist vermutlich in allen mit gesellschaftlichen Handlungsfeldern verbundenen Wissenschaften üblich. Technikwissenschaftler, Medizinerinnen, Juristen und Ökonominnen verändern die Welt dadurch, dass sie Optionen aufzeigen, die vorher nicht da waren. So entstehen neue Handlungsmöglichkeiten - und neue Optionen können sogar dann die Welt verändern, wenn sie nicht angewendet werden, etwa wenn bestehende Optionen in einem anderen Licht erscheinen. Das scheinbar in transformativer Hinsicht so unverdächtige Entwickeln von Optionen als honest broker ist so gesehen tätige Weltveränderung - und das umso mehr, weil entsprechende Argumente für ihre Leistungsfähigkeit gleich mitgeliefert werden (siehe Punkt 1).

\section{Solange die Transformationsleistung der transformativen Wissenschaft durch Forschung und argumentative Überzeugung gespeist wird und nicht aus Überredungskunst, ist sie als honest broker gleichermaßen transformativ.}

sein soll, wäre eine merkwürdige Vorstellung. Jedes Argument für die Eignung einer bestimmten Option jedoch kommt nicht ohne normative Anteile aus, ohne Kriterien für "gut" und ohne substanzielle Annahmen über die Bedeutung nachhaltiger Entwicklung (Grunwald 2016). Damit funktioniert die strenge Unterscheidung von Strunz und Gawel nicht mehr: Keineswegs legen die honest broker nur weitere Optionen auf den Tisch, die dann moderiert werden. Stattdessen bringen sie simultan Argumente bei, warum und in welcher Hinsicht die von ihnen neu entwickelten Optionen „gut“ zu sein beanspruchen. Dies ist offenkundig

1 Bei Pielke (2010, S. 17) heißt es: „One way for science advisors to closely engage with the needs of policymakers but avoid recreating themselves as special interest groups is to work to clarify and, if possible, expand the scope of choice available in decision making. (...) the Honest Broker of Policy Options seeks to explicitly integrate scientific knowledge with stakeholder concerns in the form of alternative possible courses of action."
Diese Beobachtung liefert ein weiteres Argument, warum die Rolle des honest broker mit einem Transformationsinteresse Richtung Nachhaltigkeit durchaus kompatibel ist. In vielen Feldern liegen etablierte Optionen auf dem Tisch, ob zur Energiewende, zum autonomen Fahren oder in der Chemikalienwirtschaft, die von nachhaltigkeitsmotivierten Wissenschaftler(inne)n unter Nachhaltigkeitsaspekten ausgewertet werden können. Scheidet eine Option aus, kann das dazu motivieren, neue und nachhaltigkeitsfreundlichere Optionen zu entwickeln. So wird der Optionenraum erweitert statt verengt, ganz im Sinne des honest broker. Überzeugen die neu hinzugekommenen Optionen, können sie die Welt verändern. Wenn transformative Wissenschaft dies leistet, ist sie honest broker und engagiert sich zugleich für die Nachhaltigkeitstransformation. Solange die Transformationsleistung der transformativen Wissenschaft durch Forschung und argumentative Überzeugung gespeist wird und nicht aus Überredungskunst, ist sie als honest broker gleichermaßen transformativ. 


\section{Schlussbemerkungen}

Die Argumentation von Strunz und Gawel geht also aus zwei Gründen nicht auf: Sie sehen normative Äußerungen in einem wissenschaftlichen Disput als Interessenvertretung an und sie reduzieren den honest broker auf ein welt- und wissenschaftsfremdes Konstrukt transformationsferner Moderation. Die Autoren verkennen, dass ihr Gegensatzpaar honest broker versus issue advocate zwar wunderbar illustrativ, aber analytisch nicht gerade eindeutig ist. Natürlich gilt „Engagement und Erkenntnis stehen nicht notwendigerweise in einem synergetischen Verhältnis“ (Strunz und Gawel 2017, S. 324) - aber sie stehen eben auch nicht notwendigerweise in Widerspruch zu einander! Als honest broker für die Nachhaltigkeitstransformation zu wirken, also Engagement und Erkenntnis gekonnt zu vereinbaren, ist möglich, aber auch anspruchsvoll. Insbesondere setzt es ein hohes Maß an Reflexivität und argumentativer Transparenz voraus, verlangt Lernfähigkeit nicht nur in analytisch-empirischer, sondern auch normativer Hinsicht, und ist auf das ständige Infragestellen bisheriger Positionen und damit auf den Zweifel als konstitutive Kategorie verpflichtet (Strohschneider 2014).

Man kann durchaus fragen, warum gerade die transformative Wissenschaft im Zentrum derart fundamentaler Debatten steht. Transformatives Wissen im genannten Sinne wird auch von vielen anderen Wissenschaftsbereichen erzeugt - Medizin, Technikund Rechts-, aber auch Wirtschaftswissenschaften. Dort finden keine analogen Diskussionen statt, ob die normative Ausrichtung, etwa auf Gerechtigkeit oder Gesundheit, die Erkenntnis trübt. Warum eigentlich nicht? Oder warum findet keine Debatte über die „Übergriffigkeit“ des technischen Denkens auf die Gesellschaft statt? Bereits Schelsky (1961) hat hellsichtig darüber geschrieben, wie Expertenwissen und technisches Optimierungsdenken das genuin Politische schleichend eliminieren können. Warum wird der transformativen Forschung eine „Atrophierung des Politischen“ (Strohschneider 2014) vorgehalten, aber nicht manchen Technikwissenschaften oder der Informatik?

Hier scheinen unterschwellige Sorgen eine Rolle zu spielen, die es vermutlich wert wären, aufgedeckt und offen diskutiert zu werden. Kursieren Ängste vor einer Ökodiktatur, Sorgen vor einer Verengung des Bedeutungsdiskurses zur Nachhaltigkeit (Grunwald 2016) auf das Verständnis einer bestimmten Clique? Und selbst wenn, woher käme dann die Sorge, dass eine eher kleine Bewegung so etwas bewirken könnte? Oder ist es doch die Sorge, dass transformative Wissenschaft keine Ergänzung, sondern eine Umwälzung oder gar Ersetzung des bisherigen Wissenschaftssystems erstrebt? Alle diese Sorgen liefern in der Tat beträchtlichen Zündstoff - sie sollten dann aber auch mit offenem Visier angesprochen werden.

Schließlich sei eine positive Notiz erlaubt. Es ist doch eine Freude zu sehen, wie peinlich genau und sensibel die Nachhaltigkeitsforschung und ihre Beobachter(innen) in den etablierten Disziplinen mit all diesen Fragen umgehen. Diese Reflexivität und Sorgfalt würde man sich manchmal auch von anderen transformativ wirkenden Wissenschaften wünschen - wie etwa von der Volks- wirtschaftslehre, die mit vermeintlich empirisch-analytischem Lehrbuchwissen bereits ganze Volkswirtschaften ruiniert hat.

\section{Literatur}

Gethmann, C. F., T. Sander. 1999. Rechtfertigungsdiskurse. In: Ethik in der Technikgestaltung. Praktische Relevanz und Legitimation. Herausgegeben von A. Grunwald, S. Saupe. Berlin: Springer. 117-151.

Grunwald, A. 2015. Transformative Wissenschaft - eine neue Ordnung im Wissenschaftsbetrieb? GAIA 24/1: 17-20.

Grunwald, A. 2016. Nachhaltigkeit verstehen. Arbeiten an der Bedeutung nachhaltiger Entwicklung. München: oekom.

Habermas, J. 1973. Wahrheitstheorien. In: Wirklichkeit und Reflexion. Walther Schulz zum sechzigsten Geburtstag. Herausgegeben von H. Fahrenbach, H. Pfullingen: Neske. 211-265.

Jaeger-Erben, M., E. Nagy, M. Schäfer, E. Süßbauer, J. Zscheischler. 2018. Von der Programmatik zur Praxis: Plädoyer für eine Grounded Theory transformationsorientierter Forschung. GAIA 27/1: 117-121.

Kläy, A., F. Schneider. 2015. Zwischen Wettbewerbsfähigkeit und nachhaltiger Entwicklung: Forschungsförderung braucht Politikkohärenz. GAIA 24/4: 224-227.

Ott, K., R. Döring. 2004. Theorie und Praxis starker Nachhaltigkeit. Marburg: Metropolis.

Petermann, T., H. Bradke, A. Lüllmann, M. Poetzsch, U. Riehm. 2011. What happens during a blackout? Consequences of a prolonged and wide-ranging power outage. Technology assessment studies series. Berlin: Büro für Technikfolgen-Abschätzung beim Deutschen Bundestag.

Pielke, R. A., Jr. 2007. The honest broker. Making sense of science in policy and politics. Cambridge, UK: Cambridge University Press.

Pielke, R. A., Jr. 2010. Expert advice and the vast sea of knowledge. In: Inter- und Transdisziplinarität im Wandel? Herausgegeben von A. Bogner, K. Kastenhofer, H. Torgersen. Baden-Baden: Nomos.

Rohe, W. 2015. Vom Nutzen der Wissenschaft für die Gesellschaft. Eine Kritik zum Anspruch der transformativen Wissenschaft. GAIA 24/3: 156-159.

Schelsky, H. 1961. Demokratischer Staat und moderne Technik. Atomzeitalter 5: 99-102.

Schneidewind, U. 2015.Transformative Wissenschaft - Motor für gute Wissenschaft und lebendige Demokratie. GAIA 24/2: 88-91.

Schneidewind, U., M. Singer-Brodowski, K. Augenstein, F. Stelzer. 2016. Pledge for a transformative science. Wuppertal paper 191. Wuppertal: Wuppertal Institut für Klima, Umwelt, Energie.

Strohschneider, P. 2014. Zur Politik der Transformativen Wissenschaft. In: Die Verfassung des Politischen. Festschrift für Hans Vorländer. Herausgegeben von A. Brodocz, D. Herrmann, R. Schmidt, D. Schulz, J. Schulze-Wessel. Wiesbaden: Springer. 175-192.

Strunz, S., E. Gawel. 2017. Transformative Wissenschaft - eine kritische Bestandsaufnahme der Debatte. GAIA 26/4: 321-325.

von Wissel, C. 2015. Die Eigenlogik der Wissenschaft neu verhandeln. Implikationen einer transformativen Wissenschaft. GAIA 24/3: 152-155.

Armin Grunwald

Geboren 1960 in Soest, Nordrhein-Westfalen. Promotion in Physik. Habilitation in Philosophie. Seit 1999 Leiter des Instituts für Technikfolgenabschätzung und Systemanalyse (ITAS) des Karlsruher Instituts für Technologie (KIT). Seit 2002 Leiter des Büros für Technikfolgenabschätzung beim Deutschen Bundestag. Seit 2007 Inhaber des Lehrstuhls für Technikphilosophie und Technikethik am KIT. Mitglied im Science

Committee von Future Earth und im wissenschaftlichen Beirat von GAIA Forschungsschwerpunkte: Theorie und Konzepte der Nachhaltigkeit, Technikfolgenabschätzung, Ethik der Technik 